\title{
Comparison of Two Planar Elliptical Ultra-Wideband PPy Conductive Polymer Antennas
}

\author{
Thomas Kaufmann*, Akhilesh Verma*, Said F. Al-Sarawi*, Van-Tan Truong ${ }^{\dagger}$ and Christophe Fumeaux* \\ *The School of Electrical \& Electronic Engineering, University of Adelaide, Adelaide SA, 5005, Australia. \\ Email: thomas.kaufmann@adelaide.edu.au \\ ${ }^{\dagger}$ Defence Science and Technology Organisation (DSTO) Melbourne, \\ 506 Lorimer Street, Fishermans Bend VIC 3207, Australia
}

\begin{abstract}
Conductive polymers are a new type of conductive materials that are attractive for application in flexible, and possibly reconfigurable antennas. Two planar elliptical ultrawideband antennas based on conductive polymers are manufactured. The selected Polypyrrole (PPy) polymer exhibits moderate conductivity but can be produced as rather thick free-standing films up to a size of several hundred micrometers. The efficiency of the conductive materials is estimated based on the comparison of the radiation patterns with those of a reference antenna built from copper. The two PPy samples exhibit the same conductivity, but different thicknesses. Higher efficiency is achieved using the thicker PPy sample, demonstrating efficient operation below one skin depth.
\end{abstract}

\section{INTRODUCTION}

Conductive polymers [1] are attractive materials for future applications in microwave devices due to their mechanical flexibility and ability for low-cost mass production in printing or screening processes. Additionally, the biodegradable properties of some conducting polymers make them viable for biomedical applications. Also, the ability to potentially tune the conductivity through a bias voltage is highly interesting for building tunable microwave devices. In the realm of material science, significant research efforts are currently invested into further increasing the conductivity of the polymers. At present, two prominent types of conductive polymers are Poly(3,4ethylenedioxythiophene) (PEDOT) and Polypyrrole (PPy).

This article builds on previous estimations on the efficiency of conductive polymer antennas in the microwave region of a microstrip antenna in [2] and extends the finding from [3] by comparing the conductor efficiency of two antennas over a large frequency bandwidth for different thicknesses of PPy films.

\section{Conductive Polymers: Polypyrrole}

PPy, a doped polymer, is one of the most popular conductive polymers due to its thermal and environmental stability. Both free-standing films and powder PPy can be synthesized, depending on the method of polymerization. As only a small number of samples were available, shapes were cut out with a scalpel from free-standing films.

The PPy samples were manufactured according to [4]. The films used here are 116 and $158 \mu \mathrm{m}$ thick. Using the fourprobe technique, the DC-conductivity of the film sample has been measured as almost identical $(2,720 \mathrm{~S} / \mathrm{m} @ 116 \mu \mathrm{m}$ and $2,730 \mathrm{~S} / \mathrm{m} @ 158 \mu \mathrm{m})$.

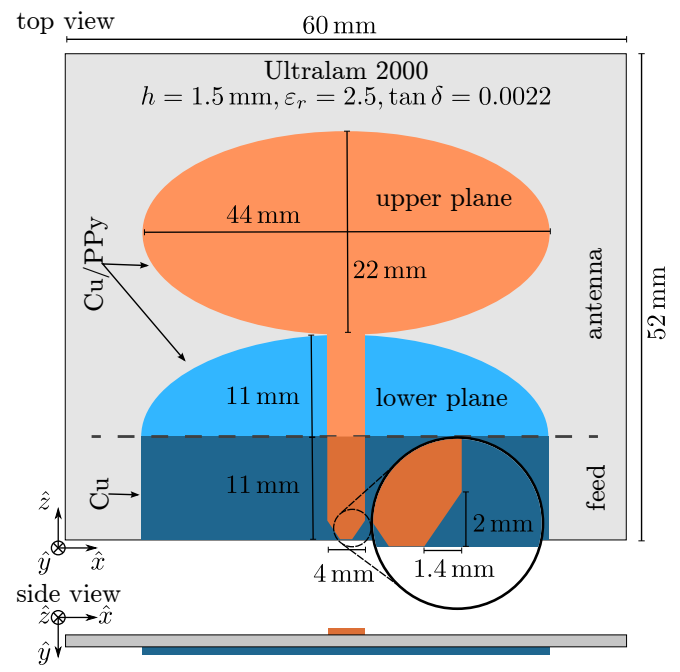

Fig. 1. Design of the UWB-Antenna with the radiating structure either manufactured in copper or PPy.

\section{ANTENNA DESIGN}

Extending the design from [5], a compact planar elliptical design has been optimized for compactness and ease of production of the radiating elements. The design is developed for an acceptable input reflection coefficient $\left(S_{11} \leq-10 \mathrm{~dB}\right)$ in the ultra-wideband (UWB) range [6]. Other relevant design criteria were robustness to manufacturing tolerances and a simple transition between the feeding and the antenna sections. The antenna consists of a microstrip feeding section and elliptical radiating elements. Fig. 1 shows the dimensions of the antenna.

For efficiency estimations, three individual antennas have been manufactured. The first has its radiating elements made from copper for reference and the other two are made of PPy films with different thicknesses. The conductors are electrically connected to the launching structure with conductive epoxy. The two different film thicknesses lead to slightly different skin depths and to different DC sheet resistances. Table I lists the estimated resistances and the skin depths at the upper and lower limits of the UWB spectrum. It can be observed that the skin depths of both PPy films are very similar. In terms of the thickness of the PPy samples both films are in the order of one skin depth, with one sample $36 \%$ thicker than the other. Hence, a better efficiency for the thicker conductor can be expected. 
TABLE I

ELECTRICAL PROPERTIES OF COPPER VS. TWO PPY FILMS.

\begin{tabular}{|c|c|c|c|c|}
\hline Material & \multirow{2}{*}{$\begin{array}{c}\text { Conductivity } \\
\text { Thickness) }\end{array}$} & \multirow{2}{*}{$\begin{array}{l}\text { DC-Sheet- } \\
\text { Resistance }\end{array}$} & \multicolumn{2}{|c|}{ Skin Depth } \\
\cline { 4 - 5 } & & $3.1 \mathrm{GHz}$ & $10.6 \mathrm{GH} z$ \\
\hline $\mathrm{Cu}(18 \mu \mathrm{m})$ & $5.8 \times 10^{7} \mathrm{~S} / \mathrm{m}$ & $10^{-3} \Omega / \mathrm{sq}$ & $1.18 \mu \mathrm{m}$ & $0.64 \mu \mathrm{m}$ \\
\hline $\mathrm{PPy}(158 \mu \mathrm{m})$ & $2720 \mathrm{~S} / \mathrm{m}$ & $2.32 \Omega / \mathrm{sq}$ & $173.3 \mu \mathrm{m}$ & $93.7 \mu \mathrm{m}$ \\
\hline $\mathrm{PPy}(116 \mu \mathrm{m})$ & $2730 \mathrm{~S} / \mathrm{m}$ & $3.16 \Omega / \mathrm{sq}$ & $173.0 \mu \mathrm{m}$ & $93.6 \mu \mathrm{m}$ \\
\hline
\end{tabular}

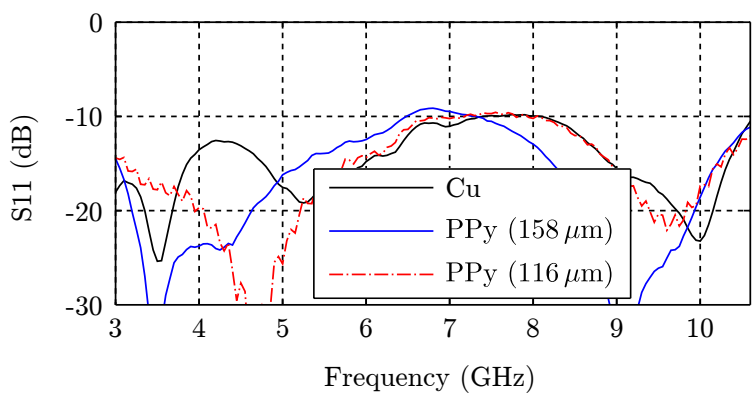

Fig. 2. Measured input reflections (S11) for the copper and the two different conductive polymer antennas in the UWB spectrum from $3.1 \mathrm{GHz}$ to $10.6 \mathrm{GHz}$.

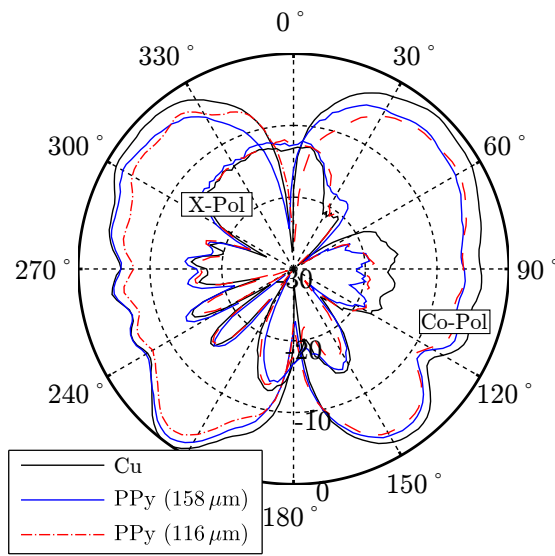

Fig. 3. Measured gain patterns in the $x z$-plane for the three antennas at $5 \mathrm{GHz}$, normalized with relation to the copper antenna.

\section{Measurements}

The input reflections (S11 parameters) for the copper and the two PPy film antennas are shown in Fig. 2. The criterion that $S 11 \leq-10 \mathrm{~dB}$ is nearly fulfilled for the whole UWB spectrum. The efficiency of the antennas relative to the copper antenna has been estimated through direct comparison of the gain patterns. Fig. 3 shows an example the normalized measured gain patterns relative to the copper antenna at $5 \mathrm{GHz}$. It can be observed that all patterns look very similar, with slightly lower gain for the conductive polymer antennas due to the conductor losses.

Through averaging of the received gain through all angles of a pattern, the conductor efficiency $e_{c}$ can be estimated according to [3] by dividing the average absolute gain of the conductive polymer antennas by the reference copper antenna. This method assumes that the copper antenna is highly efficient (close to 100\%) and allows for slight misalignments of the antennas that can occur during the measurement. Fig. 4 shows the estimated efficiencies for the two PPy film thicknesses. It can be clearly observed that both antennas

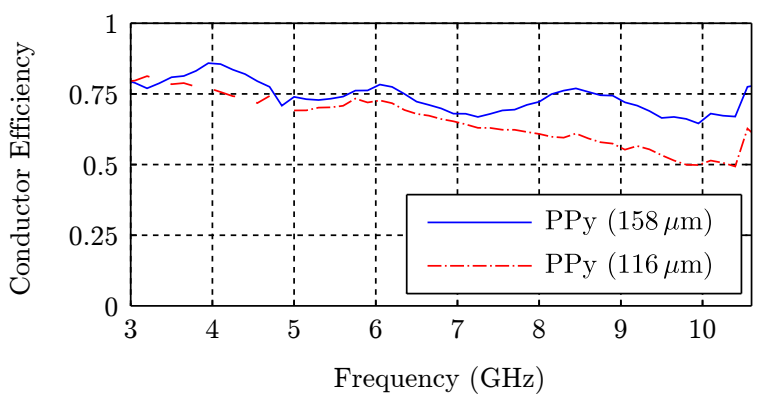

Fig. 4. Relative efficiency of the two PPy conductors in the UWB spectrum.

exhibit very satisfying efficiencies. The thicker material has an average efficiency of $e_{c}=74 \%$, while the thinner PPy film has $e_{c}=66 \%$ average efficiency over the whole UWB spectrum. At higher frequencies, the efficiency for both PPy films starts to decrease. As expected the degradation is more pronounced for the thinner film.

\section{CONCLUSION}

The efficiency of planar elliptical ultra-wideband antennas with radiating elements fabricated from conductive polymers (PPy) has been estimated. Measurements have shown very similar radiation patterns for a copper reference antenna and two antennas based on PPy. It has been shown that for two different PPy films with a thickness at the limit of their skin depth, realized antennas can exhibit a very satisfying conductor efficiency, with an advantage for the thicker film. These results demonstrate the importance of appropriate film thickness for antenna applications using less conductive materials, and ultimately illustrate the feasibility of applying conductive polymers in the microwave region as promising conductor materials with plastic-like mechanical properties.

\section{ACKNOWLEDGEMENT}

The authors acknowledge the support of the Australian Research Council (ARC) under Discovery Project DP120100661.

\section{REFERENCES}

[1] C. K. Chiang, C. R. Fincher, Y. W. Park, A. J. Heeger, H. Shirakawa, E. J. Louis, S. C. Gau, and A. G. MacDiarmid, "Electrical Conductivity in Doped Polyacetylene," Phys. Rev. Lett., vol. 39, pp. 1098-1101, Oct 1977. [Online]. Available: http://link.aps.org/doi/10.1103/PhysRevLett.39.1098

[2] A. Verma, C. Fumeaux, V.-T. Truong, and B. Bates, "Effect of film thickness on the radiation efficiency of a $4.5 \mathrm{GHz}$ polypyrrole conducting polymer patch antenna," in Proc. IEEE Asia-Pacific Microwave Conference (APMC) 2010, December 2010, pp. 95-98.

[3] T. Kaufmann, A. Verma, V.-T. Truong, B. Weng, R. Shepherd, and C. Fumeaux, "Efficiency of a compact elliptical planar ultra-wideband antenna based on conductive polymers," International Journal of Antennas and Propagation, 2012, submitted.

[4] V.-T. Truong and J. G. Ternan, "Complex conductivity of a conducting polymer composite at microwave frequencies," Polymer, vol. 36, no. 5, pp. 905-909, 1995. [Online]. Available: http://www.sciencedirect.com/science/article/B6TXW3YTJTFF-92/2/0395a2f6b3e3a2bacc825ccf42d1f8d0

[5] K. C. L. Chan, Y. Huang, and X. Zhu, "A planar elliptical monopole antenna for UWB applications," in Proc. IEEE/ACES Int. Wireless Communications and Applied Computational Electromagnetics Conf, 2005, pp. 182-185.

[6] H. Schantz, The Art and Science of Ultrawideband Antennas. Artech House, 2005. 